\title{
Valor Agregado y Nivel Competitividad de las pymes en el Cantón Machala, Ecuador.
}

\section{Value Added and Competitiveness Level of Pymes in the Machala, Ecuador}

Armando Urdaneta. ${ }^{1}$, Andreina Inés González Ordóñez. ${ }^{2}$, Laura Rosa Luciani Toro ${ }^{3}$. \& Emmanuel Borgucci. ${ }^{4}$

DOI: https://doi.org/10.33262/visionariodigital.v3i2.422

\section{Resumen.}

El artículo tiene como objetivo determinar la relación entre el valor agregado y nivel competitividad de las pequeñas y medianas empresas (Pymes) en el cantón Machala, Provincia de El Oro. Para ello se consideraron los indicadores de fortalezas y debilidades: planificación, calidad, recurso humano, gestión ambiental, comercialización, administración, contabilidad y finanzas; todos ellos, esbozados en el mapa de competitividad del BID. Se realizó una investigación descriptiva y transeccional, cuya muestra fue de 217 pymes de una población de 1406 de los sectores comercio, servicio y agropecuario del cantón Machala. Los resultados evidencian que el cantón Machala debe continuar profundizando su proceso de diversificación económica y aumento de su participación en la economía regional mediante un crecimiento sostenido a los efectos de aprovechar sus potencialidades humanas y materiales. En consecuencia, es necesario que los números de Pymes sigan creciendo y sobre todo mejoren sus procesos de trabajo en lo atinente al manejo de la gestión ambiental, la gestión del recurso desarrollo de una planificación estratégica que les permita responder a un plan estratégico.

Palabras clave: Valor Agregado, Competitividad, Pymes

\section{Abstract}

This article aims to determine the relationship between the added value and competitiveness level of small and medium enterprises (SMEs) in the Machala Canton,

\footnotetext{
1 Universidad Metropolitana, Machala, Ecuador, aurdaneta@umet.edu.ec

2 Universidad Metropolitana, Machala, Ecuador, aigonzalez@umet.edu.ec

3 Universidad Metropolitana, Machala, Ecuador, 1luciani@umet.edu.ec

4 Universidad del Zulia, Maracaibo, Venezuela, emmanuelborgucci@gmail.com
} 
El Oro province. For this, indicators of strengths and weaknesses were considered: planning, quality, resource human, environmental management, marketing, administration, accounting and finance; all of them, outlined in the competitiveness map of the IDB. A descriptive and transactional investigation was carried out, whose sample was of 217 SMEs from a population of 1406 from commerce, service and agricultural sectors of the Machala Canton. The results show that the Machala Canton must continue to deepen its process of economic diversification and increase its participation in the regional economy through sustained growth in order to take advantage of its human and material potential. Consequently, it is necessary that the number of SMEs continue to grow and, above all, improve their work processes in relation to the management of environmental management, resource management, development of a strategic planning that allows them to respond to a strategic plan.

Key words: Added Value, Competitiveness, SMEs.

\section{Introducción.}

Las empresas de la Provincia de El Oro representan el 5,1\% del total de empresas del Ecuador, del total de 41.643 empresas, el 99,7\% corresponde a pymes, de este porcentaje, $89,5 \%$ corresponde a microempresas, el $8,4 \%$ a pequeñas empresas y el 1,8\% a medianas empresas (INEC, 2015). Asimismo, la actividad productiva de la provincia se concentra (65\%) en tres cantones: Machala (45\%), Pasaje (10\%) y Santa Rosa (10\%), de los catorce que posee la provincia. En la mayoría de los cantones los sectores comercio, servicios y agroindustria son los más importantes. En este sentido, en la provincia el $41 \%$ de las empresas está dedicado al comercio, el $34 \%$ se dedica al sector servicios y el $15 \%$ lo conforma la agroindustria.

Es importante el estudio de la pyme en el Ecuador, tal como lo indican Bermejo \& Saavedra (2018) debido a su destacada participación en el desarrollo económico del país, siendo por ello primordial fortalecer este sector, porque muchas empresas muestran un sistema productivo, administrativo, financiero y tecnológico primitivo lo que afecta principalmente a su competitividad, según los referidos autores. Por otra parte, destacan los autores, resulta preocupante ver la velocidad con la que aparecen o desaparecen posiblemente por la poca capacidad de respuesta a las realidades cambiantes de tipo económico, político, social y cultural que se suceden en un mundo tan globalizado como en el que hoy se encuentra inmerso.

Como resultado de la ejecución del proyecto de investigación titulado Propuestas de mejoras para la competitividad interna empresarial de las pymes de la provincia de El Oro, el cual es desarrollado por la carrera de Gestión Empresarial de la UMET, sede Machala, se presenta el siguiente artículo que tiene como objetivo determinar la relación entre el valor agregado y nivel competitividad de las Pymes en el cantón Machala; en aras de analizar cómo aquellas economías cantonales, como la sujeto a estudio, donde hay una mayor presencia pequeñas y medianas empresas, sus expectativas de crecimiento económico a largo plazo son mucho más factibles que a nivel nacional donde hay una menor presencia de Pymes, por lo que su participación en el valor agregado cantonal total, muestra una tendencia creciente. 
No obstante, se hace necesario considerar una serie de indicadores concebidos en la metodología del mapa de competitividad del BID, como planificación, calidad, recurso humano, gestión ambiental, comercialización, administración, contabilidad y finanzas que permitan medir las fortalezas y debilidades internas de las pymes con la finalidad de mejorar las áreas de las mismas que puedan estar afectando su nivel de competitividad.

\section{Desarrollo.}

$\mathrm{Al}$ relacionar las pequeñas y medianas empresas y el desarrollo económico, para Gallino (2003), no existe una significativa influencia de la pequeña y mediana empresa en el desarrollo de la estructura productiva de Italia. Al contrario, el autor subraya el hecho de que es la gran empresa la que sabe aprovechar los recursos humanos y tecnológicos para realizar grandes innovaciones. Esto último gracias a una intensa actividad de investigación, que requieren ingente cantidad de recursos. Por su parte Onida (2004), sostiene que existe una relación indisoluble entre la innovación y la investigación científica. Lo curioso es que la demanda de innovaciones proviene principalmente de pequeñas empresas que desarrollan sus productos y procesos in house. Este autor dice que existen países en que este proceso no se presenta de manera extendida y es el estado quien realiza la demanda de innovaciones, lo que se traduce en un aumento de las importaciones de tecnología. Estaos puntos de vista son compartidos por Nardozzi (2004), Rossi (2006) y Alesina e Giavazzi (2006).

Dentro de la llamada hipótesis de las pequeñas y medianas empresas como un valor y recurso distintivo, Ghiringhelli y Pero (2010), destacan la capacidad de las empresas de este tipo para sortear coyunturas económicas desfavorables, así como afrontar grandes transformaciones de manera creativa. Por otra parte, al menos para el caso de Italia, la pequeña y mediana empresa ha contribuido a la expansión de la fuerza de trabajo (Preti y Puricelli, 2007). Sin embargo, las pequeñas y medianas empresas necesitan para su desarrollo el concurso o las relaciones estables con las grandes corporaciones industriales (De Rita, 2006). Es decir, surge la llamada hipótesis de la complementariedad entre las pequeñas y medianas empresas y las grandes empresas (L'ipotesi della complementarietá tra PMI e grandi imprese).

Para los efectos del presente estudio, se consideraron una serie de trabajos de investigación previos, como el desarrollado por Astudillo y Briozzo (2016) titulado Innovación en las pymes manufactureras de Ecuador y Argentina el cual analiza las características de las micro, pequeñas y medianas empresas manufactureras de países incluidos en la base de datos de Enterprise Survey del año 2010 del Banco Mundial. Este trabajo muestra que las empresas manufactureras argentinas son más propensas en innovar en productos y procesos respectos a las del Ecuador. Aunque actividades de innovación como el gasto en investigación y desarrollo sobre ventas, registro de patentes y apoyo del sector público presentan semejanzas entre ambos países.

En el caso de Ecuador, el análisis evidencia una menor propensión a innovar en el producto y en el proceso comparado con Argentina, debido a los obstáculos que los 
empresarios encuentran por la falta de políticas públicas. Al respecto, Zamora y Villamar (2011) señalan en su análisis sobre las pymes manufactureras en Quito-Ecuador que no realizan actividades de innovación por las limitaciones del orden económico, falta de incentivos fiscales para desarrollar ciencia y tecnología, así como la falta de información para acceder a programas de fomento, servicios y centros de desarrollo sobre innovación, limitaciones de la capacidad tecnológica, entre otros.

Asimismo, Yance et al. (2017), en su investigación titulada "La importancia de las Pymes en el Ecuador" señalan que en la actualidad las Pymes representan la mayor fuerza económica del país. La globalización y los rápidos cambios en la tecnología, imponen enormes retos a las empresas para crecer y afrontar con éxito los nuevos desafíos y asegurar su permanencia en los mercados. Las Pymes según Yance et al. (2017) se caracterizan por ser administrada por una persona natural o jurídica y que realizan algún tipo de actividad dentro del sistema productivo del país, que comercializan bienes o servicios.

Según la Ley de Compañías mediante el artículo 428, en Ecuador, se establece que las Pymes pueden ser asociaciones de formalidades. Y a su vez exige que todas estas unidades de negocio deben cumplir con lo establecido en la Ley de Régimen Tributario Interno y RUC". (Superintendencia de Compañías, 2012)"

En ese orden de ideas las Pymes se pueden clasificar en:

Tabla 1 Clasificación de las empresas según su tamaño

\begin{tabular}{lll}
\hline Tipo de empresa & Personal ocupado & Valor Bruto de las ventas anuales (US\$) \\
\hline Microempresa & 1 a 9 & $\leq 100.000$ \\
Pequeña empresa & 10 a 49 & $100.001-1.000 .000$ \\
Mediana empresa (A) & 50 a 99 & $1.000 .0001-2.000 .000$ \\
Mediana empresa (B) & 100 a 199 & $2.000 .001-5.000 .000$ \\
Grande empresa & Más de 200 & Más de 5.000.000 \\
\hline
\end{tabular}

Fuente: Instituto Nacional de Estadística y Censos (2014).

Entre los aportes resaltantes, aunado a lo ya referido, es que las Pymes representan $44 \%$ del total de las empresas establecidas, significando 84,3\% de los establecimientos, $37,7 \%$ del personal ocupado y $13 \%$ de las aportaciones al PIB, según cifras del Instituto Nacional de Estadística y Censos (2014); de las cuales 63,16\% pertenecen al sector servicios, $25,39 \%$ a la actividad comercial, 5,42\% al ramo de manufactura, 5,31\% al sector agrícola y $0,72 \%$ a la explotación minera, todo ello según cifras de la Superintendencia de Compañías (2012).

Ante el escenario descrito, para las Pymes en el Ecuador resulta sumamente importante elevar sus niveles de competitividad. En ese sentido, Saavedra (2012), en su trabajo, una 
propuesta para la determinación de la competitividad en la pyme latinoamericana establece como objetivo, proponer una metodología que permita determinar el nivel de competitividad de las pymes, identificando por áreas el nivel en que se encuentra la misma, considerando criterios tanto internos como externos; Siguiendo la metodología de mapas de competitividad del Banco Interamericano de Desarrollo (BID), el cual incluye factores tales como: Planeación estratégica, producción y operaciones, calidad, comercialización, contabilidad y finanzas, recursos humanos, gestión ambiental y sistemas de información, y complementada con indicadores externos basados en el enfoque de competitividad sistémica.

Dicha investigación concluye la conveniencia de manejar la competitividad empresarial considerando los aspectos del entorno que afecta a la organización. Por lo cual la propuesta metodológica para la determinación de la competitividad empresarial que considera tanto los factores internos de la pyme, utilizando el mapa del BID, como sus factores externos, utilizando el enfoque de Competitividad Sistémica de la CEPAL (Comisión Económica para América Latina y El Caribe) es de alta pertinencia.

Asimismo, se tomaron un conjunto de teorías fundamentales para sustentar las variables de investigación; las cuales fueron estudiadas tomando en consideración el objetivo general del Proyecto para el fomento de las MIPYMES Ecuatorianas elaborado por la secretaría de MIPYMES y Artesanías a través del Ministerio de Industrias y Productividad en febrero de 2013; el cual plantea Mejorar la productividad y competitividad de micro, pequeñas y medianas empresas de forma individual $o$ asociadas, para que sus productos accedan a los mercados nacionales e internacionales.

Este objetivo se desarrolla a través de un conjunto de objetivos específicos que resultan también de alta pertinencia para la presente investigación, como es el de implementar programas de mejoramiento en gestión empresarial y en los procesos de producción para el incremento en la productividad de las MIPYMES y fortalecer los mecanismos en la cadena de valor. Entendiendo la cadena de valor según Sánchez (2008, pág. 34) como la herramienta principal de análisis estratégico de costos de un negocio; mediante la identificación de las actividades, funciones y procesos de trabajo que se ejecutan durante el diseño de la producción, la comercialización, la entrega y el soporte de un producto o servicio.

Mientras que el valor agregado según De Gregorio (2012: 22) lo define como el valor bruto de la producción menos las compras intermedias; donde el valor total de la producción, incluyendo los insumos intermedios, se llama valor bruto de la producción, y las compras intermedias corresponde a la inversión en insumos para la producción más el costo de depreciación de los bienes de capital. De allí la importancia de la competitividad, según Dussel (2001) como procesos de integración dinámica de países y productos a mercados internacionales, dependiendo de las condiciones de oferta y demanda; siendo por ello un elemento fundamental que tiene como premisa elevar la productividad de las empresas, en especial de las Pymes, de esa forma incrementar el nivel de valor agregado. 
En el caso del valor agregado de Machala al representar las micro, pequeñas y medianas empresas 99,49\% de las organizaciones establecidas en dicho cantón y constituir según proyecciones en base a datos del INEC (Instituto Nacional de Estadística y Censo, 2017) el $60,18 \%$ de las ventas y en similar cuantía el valor agregado, resulta que no puede pretermitir elevar competitividad y con ello la productividad, mediante el mejoramiento de sus procesos de trabajo en ocho áreas fundamentales expuestas en la metodología del mapa de competitividad del BID (Banco Interamericano de Desarrollo), estas son: planificación, comercialización, administración, contabilidad y finanzas, recursos humanos, calidad, gestión ambiental y recursos humanos, Saavedra (2012:118-119).

Ahora bien, la presente investigación propone un esquema de trabajo conjuntamente con las ochos áreas fundamentales expuestas en la metodología del mapa de competitividad del BID (Banco Interamericano de Desarrollo), a través de desarrollo de un plan estratégico que comporte la siguientes etapas, fundamentado en el proceso de elaboración del plan estratégico, planteado por Sainz de Vicuña (2010: 59).

Figura 1 Modelo de Plan Estratégico

\begin{tabular}{|c|c|c|c|c|c|}
\hline $\begin{array}{c}\text { Misión y } \\
\text { visión de la } \\
\text { organización }\end{array}$ & $\begin{array}{c}\text { Antablecimie } \\
\text { nto de }\end{array}$ & $\begin{array}{c}\text { Definición de } \\
\text { los Planes de } \\
\text { acción para } \\
\text { el logro de } \\
\text { los objetivos a } \\
\text { corto y } \\
\text { largo plazo }\end{array}$ & $\begin{array}{c}\text { Asiganción } \\
\text { de recursos } \\
\text { técnicos, } \\
\text { económicos y } \\
\text { operativos } \\
\text { para el logro } \\
\text { de los } \\
\text { objetivos }\end{array}$ & $\begin{array}{c}\text { Desarrollo } \\
\text { de un plan } \\
\text { de control y } \\
\text { seguimieto al } \\
\text { plan }\end{array}$ \\
estratégico
\end{tabular}

Fuente: Elaboración propia (2019)

Figura 2 Proceso de Elaboración del Plan Estratégico

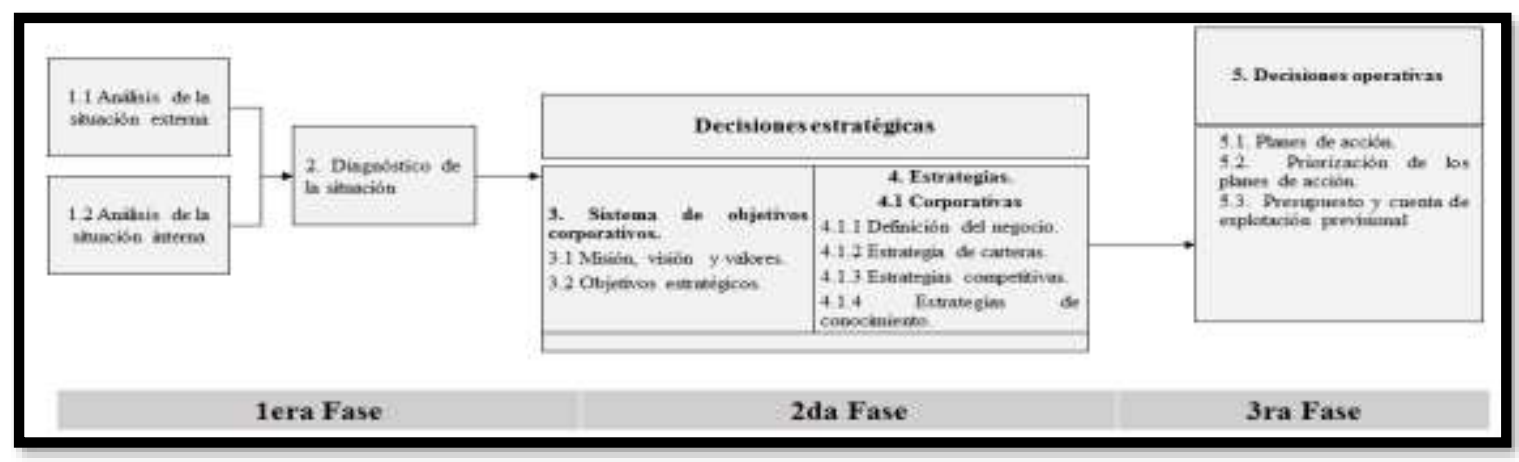

Fuente: Sainz de Vicuña (2010: 59)

El plan estratégico de una empresa, es el plan maestro en el que la alta dirección recoge las decisiones estratégicas corporativas que ha adoptado hoy, es decir en el momento que ha realizado la reflexión estratégica con su equipo, en referencia a lo que hará en los tres próximos años (horizonte habitual), para lograr una empresa competitiva que le permita satisfacer expectativas de sus diferentes grupos de interés (Sainz de Vicuña, 2010: 59). 
En ese sentido el presente estudio puntualiza las herramientas del análisis FODA (Fortaleza, Oportunidades, Debilidades y Amenazas); CAME (Corregir, Afrontar, Mantener y Explotar), como la piedra angular para la construcción del modelo de plan estratégico propuesto por los investigadores, donde los objetivos a corto y largo plazo se establecen en función de las estrategias cruzadas del análisis CAME, mediante estrategias de reorientación, supervivencia, defensivas y ofensivas, pero que parten de la definición de las fortalezas, oportunidades, debilidades y amenazas, planteadas en el FODA.

El análisis FODA según Zambrano (2011: 84) constituye otra herramienta o técnica utilizada en el momento I del modelo de gerencia estratégica. Al igual que el árbol de competencias, factores críticos de éxito y capacidades y habilidades clave, por lo que compone también una técnica empleada en la planificación corporativa, válida para las organizaciones privadas y públicas, la cual facilita la evaluación situacional de las mismas, y determina los factores que influyen y exigen desde el exterior hacia la institución.

Esos factores son externos cuando se convierten en amenazas u oportunidades que condicionan, en mayor o menor grado, el desarrollo o alcance de la misión, la visión, los objetivos y metas de la organización. Igualmente pueden ser factores internos, si se trata de fortalezas y debilidades internas, lo cual permite precisar condiciones en las que se encuentra la organización con relación a determinados objetivos o metas que se hayan planteado, según Zambrano (201:84).

El Análisis CAME es una metodología suplementaria a la del Análisis DAFO, que da pautas para actuar sobre los aspectos hallados en los diagnósticos de situación obtenidos anteriormente a partir de la matriz DAFO. Normalmente las estrategias generadas por el análisis CAME se pueden enmarcar en uno de estos grupos (www.pdcahome.com):

Estrategias defensivas (Amenazas y Fortalezas): Buscan evitar que empeore la situación actual (evitar perder cuota de mercado, etc.). En estas estrategias predominarán las acciones para en afrontar amenazas y mantener fortalezas.

Estrategias ofensivas (Fortalezas y Oportunidades): Buscan mejorar la situación actual (ganar cuota de mercado, etc.). En estas estrategias predominarán las acciones para explotar las oportunidades y mantener/reforzar las fortalezas.

Estrategias de reorientación (Debilidades y Oportunidades): Busca trasformar la situación haciendo cambios que eliminen nuestras debilidades y creen nuevas fortalezas. En este tipo de estrategias predominarán las acciones enfocadas a corregir debilidades y explotar oportunidades.

Estrategia de supervivencia (Debilidades y Amenazas): Busca eliminar los aspectos negativos que nos perjudican. En este tipo de estrategias predominarán las acciones enfocadas a corregir las debilidades y a afrontar amenazas. 
Figura 3 Análisis FODA y CAME

\begin{tabular}{|c|c|c|c|}
\hline \multicolumn{4}{|c|}{ RELACION ENTRE ANALISIS CAME Y DAFO } \\
\hline & \multicolumn{3}{|c|}{ ANALISIS EXTERNO } \\
\hline \multirow{3}{*}{ 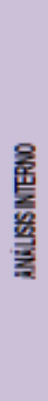 } & DAFO & OPORTUNIDADES & AMENAZAS \\
\hline & $\frac{\text { 䒿 }}{\text { 总 }}$ & $\begin{array}{l}\text { CAME: EXPLOTAR OPORTUNIDADES (E) } \\
\text { ESTRATEGIAOFENSIVA (ATADUE- } \\
\text { POSICIONAMIENTO) } \\
\text { POTENCIAR } \\
\text { mverzir: definir lacorrespondencia intre } \\
\text { fortaiezas yoportunidades quenos ileve a } \\
\text { ventajas comparativas. }\end{array}$ & $\begin{array}{l}\text { CAME: MANTENER FORTALEZAS (M) } \\
\text { ESTRATEGIA: DEFENSIVA } \\
\text { EVALUAR EL RIESGO } \\
\text { Defender: indican una necesidad de movilizar los } \\
\text { recursos }\end{array}$ \\
\hline & $\begin{array}{l}\text { 总 } \\
\text { 总 } \\
\text { 鼌 }\end{array}$ & $\begin{array}{l}\text { CAME: CORREGIR DEBILIDADES (C) } \\
\text { ESTRATEGIA: REORIENTACION } \\
\text { TOMAR DECISIONES } \\
\text { Decidir: requieren una decisión: invertir. } \\
\text { retirar, colaborar... }\end{array}$ & $\begin{array}{l}\text { CAME: AFRONTAR AMENAZAS (A) } \\
\text { ESTRATEGIA: SUPERVIVENCIA } \\
\text { CONOCERLAS LIMITACIONES } \\
\text { Controlar dianos: es neCesario controlarlos } \\
\text { DErjuicios. }\end{array}$ \\
\hline & & 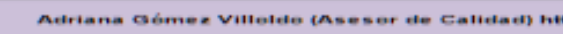 & 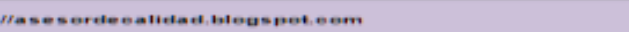 \\
\hline
\end{tabular}

Fuente:http://asesordecalidad.blogspot.com/2017/02/analisis-came-herramientade.html\#.XFDbF1VKjIU

\section{Método}

El presente estudio se fundamenta en un enfoque descriptivo y cuantitativo tal como lo indica Hernández et al (2014). Se siguió un diseño de investigación de tipo transeccional descriptivo (Hernández et al, 2014), con el objetivo de indagar la incidencia de las modalidades o niveles de una o más variables en una población. El procedimiento consiste en ubicar en una o diversas variables a un grupo de personas u otros seres vivos, objetos, situaciones, contextos, fenómenos, comunidades, etc., y proporcionar su descripción.

Para extrapolar a poblaciones muy grandes se utiliza la formula $N=\frac{Z^{2} p q}{e^{2}}$ donde el valor de $Z$ correspondiente al nivel de confianza del 95\% (expresado también así: $\alpha=0.05$ ) corresponde a $\mathrm{Z}=1,96$ sigmas o errores típicos; $\mathrm{Z}=2$ (dos sigmas) corresponden a un 95,5\% (aproximadamente, $\alpha=0.045$ ). Donde pq representa la varianza de la población, partiendo del supuesto que $\mathrm{p}=\mathrm{q}=0,5$, con lo cual $\mathrm{pq}=0,25$ el cual es válido para calcular el tamaño de la muestra, aun cuando las preguntas no sean dicotómicas. Por otra parte, el error muestral se representa con la letra e, que significa error o desviación posible cuando extrapolamos los resultados, es decir el margen de error que aceptamos. Para los efectos del presente estudio se trabajó con un nivel de confianza del $95 \%$ y admitiendo un margen de error del 9\%; donde $\mathrm{pq}=0,25$.

$$
N=\frac{1,96^{2} * 0,25}{0,09^{2}}=\frac{3.84 * 0,25}{0,0081}=118,51 \approx 119
$$

La cifra anterior indica que con la muestra seleccionada de 127 Pymes para este estudio es suficiente.

\section{Resultados}

Los resultados de la presente investigación pretenden constatar la relación intrínseca entre la evolución del entorno macroeconómico y el nivel de competitividad de la mediana y 
pequeña empresa, realizando un análisis descriptivo de las actividades económicas del cantón Machala, la participación de estas en el valor agregado total y su tasa de crecimiento promedio anual, describir el valor agregado total del cantón Machala, y el valor agregado total cantonal de la economía ecuatoriana, así como el nivel de participación del primero en el segundo.

Gráfico 1 Actividades económicas en el cantón Machala-provincia El Oro, Ecuador

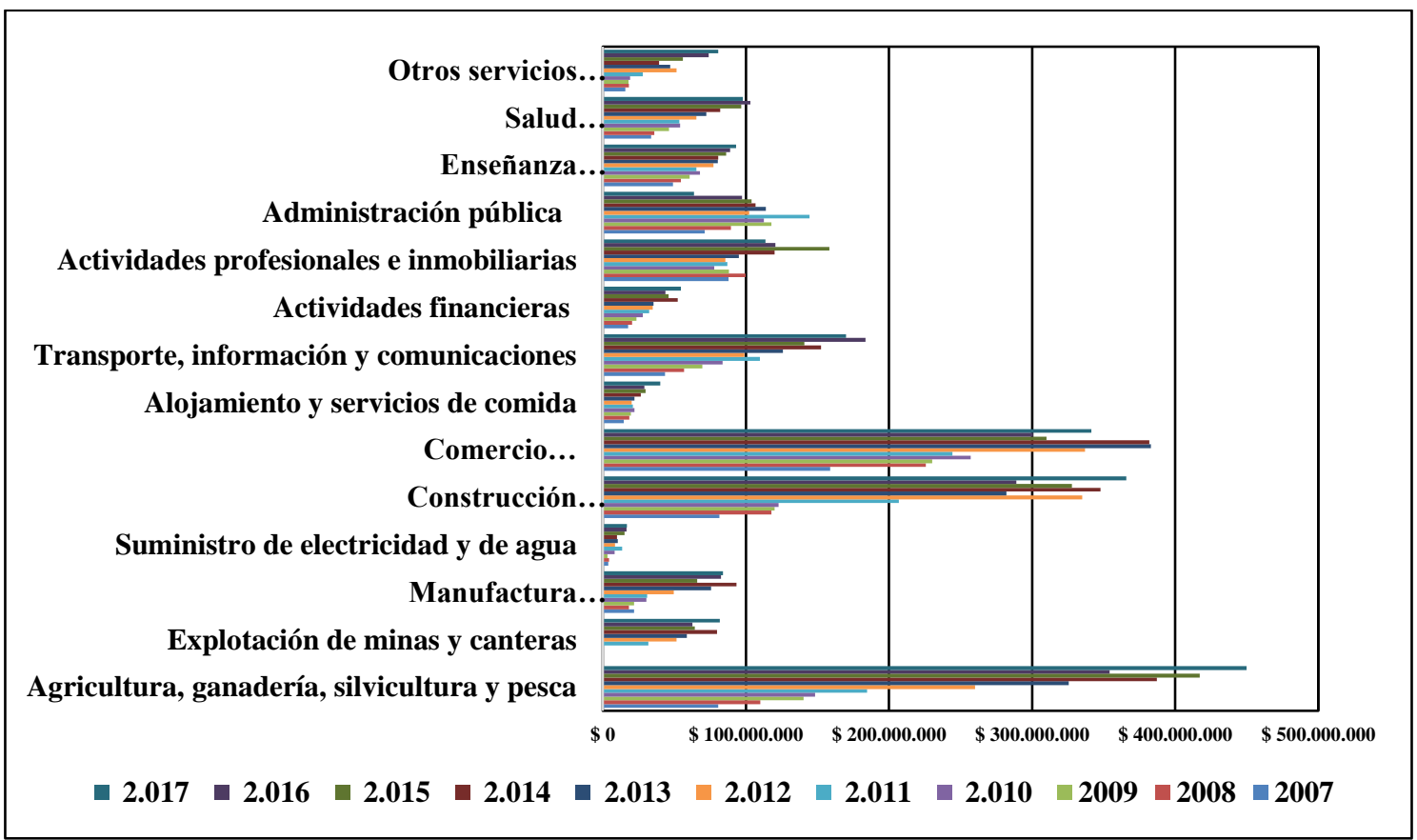

Fuente: Banco Central del Ecuador (2018)

En el gráfico 1 se muestra la evolución en términos absolutos de las diferentes actividades económicas en el cantón Machala en la provincia del Oro, Ecuador, siendo la agricultura, ganadería, silvicultura y pesca la principal actividad económica cuyo ejercicio supera los 400 millones de dólares anuales, luego le siguen comercio y construcción, las cuales despuntan sobre los 300 millones de dólares, mientras el resto de sectores económicos no superan los 200 millones de dólares a lo largo del período analizado, ello deja entrever dónde están las fortalezas y potenciales productivas del cantón Machala.

No obstante es importante resaltar que actividades económicas tan importantes como la manufactura y las actividades financieras para el desarrollo de un país no superan los 100 millones de dólares, y se encuentran poco avanzadas alcanzando su tope máximo en el año 2014 de 52 millones de dólares para la actividades financieras y 94 millones de dólares para el sector manufactura; sin embargo ambos han mostrado tasas promedio de crecimiento interanual de 12,98\% y 17,79\% respectivamente; por debajo de la agricultura, ganadería, silvicultura y pesca con 19,88\%, construcción 19,56\% y comercio 9,38\%; la cual a pesar de ser la actividad económica de mayor consolidación como se muestra en el gráfico número 2, al representar el $20 \%$ del valor agregado total acumulado de la 
economía en lapso estudiado, seguida por agricultura, ganadería, silvicultura y pesca con $18 \%$ y comercio $16 \%$.

Gráfico 2 Participación en el valor agregado total y tasa de crecimiento promedio anual en el cantón Machala-provincia El Oro, Ecuador

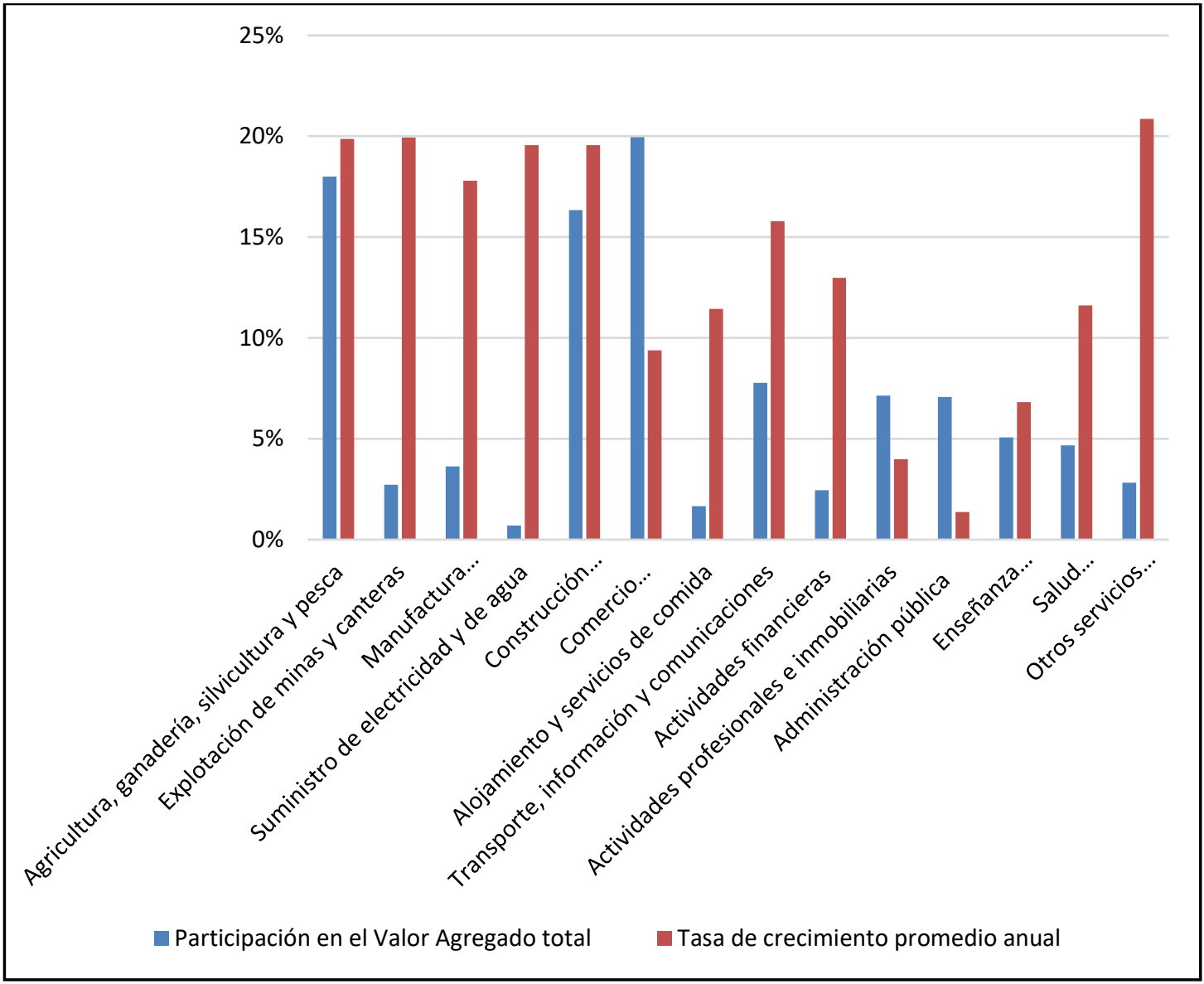

Fuente: Banco Central del Ecuador (2018)

Al analizar concienzudamente el gráfico 2 se evidencia que las actividades económicas Explotación de minas y canteras, manufactura y suministro de electricidad; aunque tienen una baja participación en el valor agregado total acumulado, presentan una alta tasa de crecimiento promedio anual en los últimos 10 años que oscila entre un $18 \%$ y 20\%; al igual que otros servicios que alcanzó una tasa de crecimiento promedio anual de $21 \%$; no así actividades como servicios profesionales, administración pública y enseñanza los cuales presentan una baja participación en el valor agregado total acumulado y una baja tasa de crecimiento promedio anual que solo una de ellas apenas supera $5 \%$.

Mientras actividades como agricultura, ganadería, silvicultura y pesca al igual que construcción tienen como característica común alta participación en el valor agregado total acumulado y una alta tasa de crecimiento promedio anual que alcanza a $20 \%$. A diferencia de comercio la cual a pesar de tener una alta participación en el valor agregado total acumulado, presenta una baja tasa de crecimiento promedio anual que apenas llega a 9\%. Sumado a ello otras actividades económicas transporte, información y comunicación, actividades financieras, enseñanza y salud presentan tasas de crecimiento 
promedio anual superiores a su tasa de participación en el valor agregado total del cantón Machala.

Estas elevadas tasas de crecimiento constatan el crecimiento sostenido que ha tenido el valor agregado total de la economía en el cantón Machala en la provincia de El Oro; mostrando una tasa de crecimiento promedio interanual de $6,69 \%$, la cual resulta al dividir la función marginal entre la función total de la línea de tendencia mostrada para el gráfico 3, la cual explica el $94 \%$ de la varianza del valor agregado total de la economía en el cantón Machala en la provincia de El Oro. Así mismo la serie de tiempo mostrada en gráfico 3 para el período 2007-2017 muestra un componente de tendencia ascendente debido a que la pendiente de la función es positiva, al igual que un componente cíclico, que oscila por debajo de la línea de tendencia en el lapso 2007-2011 y 2015-2017; por encima de la línea de tendencia en el lapso 2012-2015.

Gráfico 3 Valor agregado total de la economía en el cantón Machala en la provincia El Oro

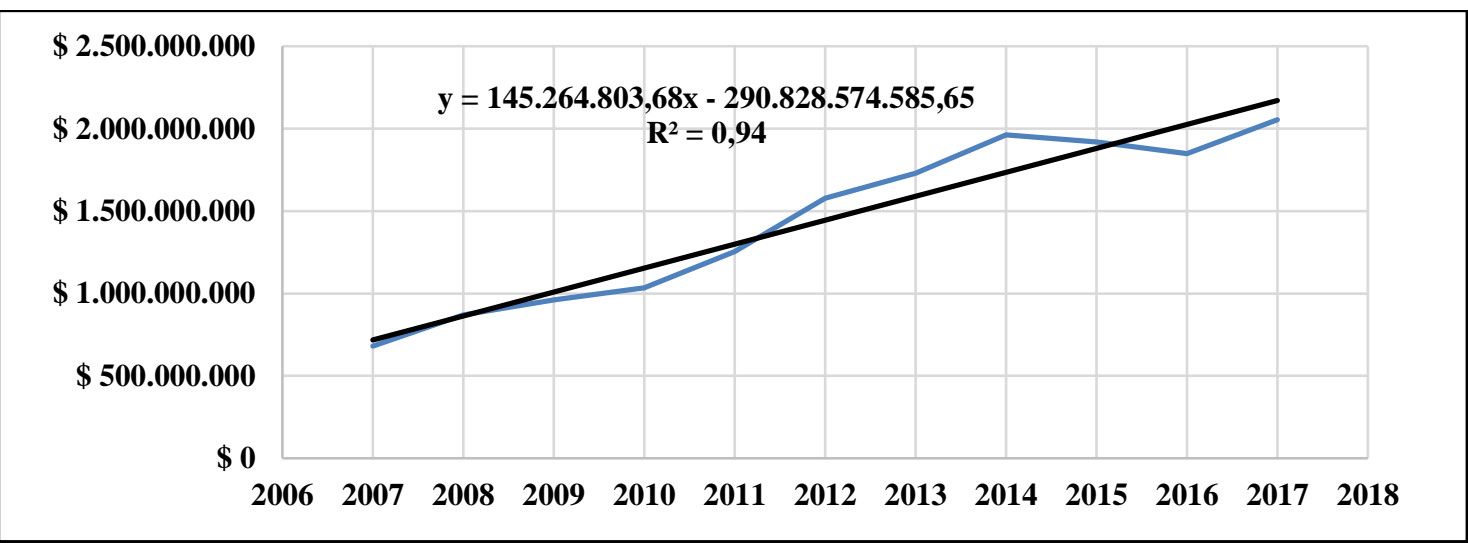

Fuente: Banco Central del Ecuador (2018)

Gráfico 4 Participación del valor agregado del cantón Machala en el valor agregado cantonal total

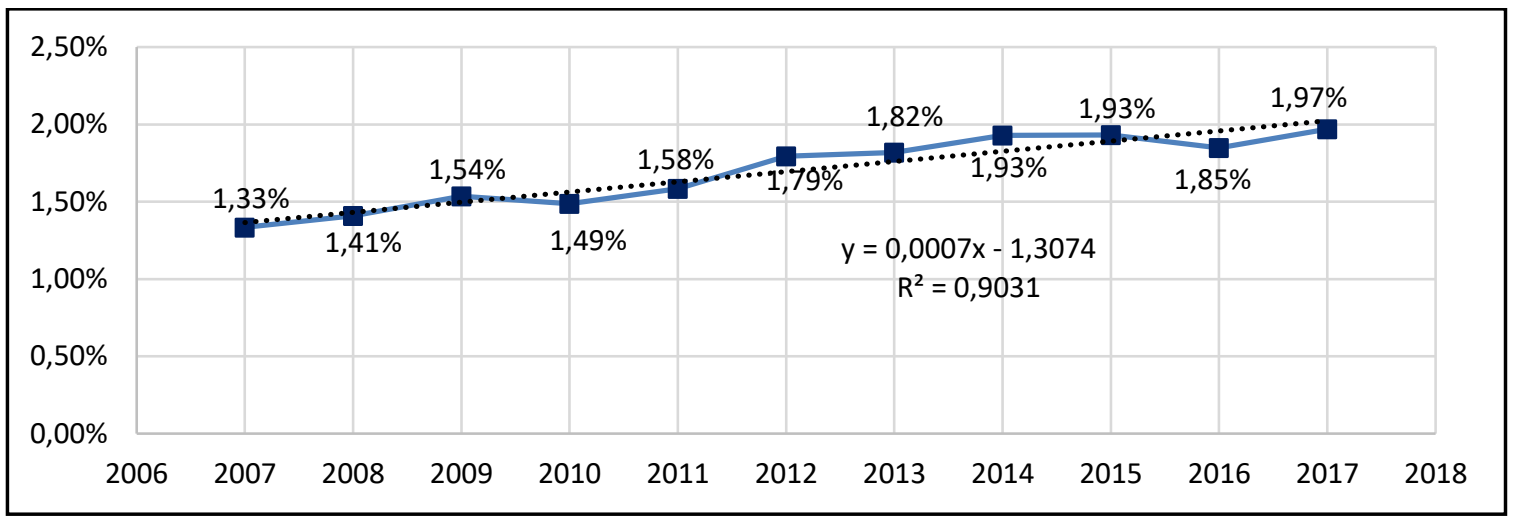

Fuente: Banco Central del Ecuador (2018)

El gráfico 4 muestra la participación del valor agregado del cantón Machala en el valor agregado cantonal total, evidencia un crecimiento interanual de la tasa de participación 
del cantón Machala en el valor agregado total de la economía Ecuatoriana de 0,07\%, la cual resulta al dividir la función marginal entre la función total de la línea de tendencia mostrada para el gráfico 3; tal escenario ha permitido que la tasa de participación pase de $1,33 \%$ en 2007 a 1,97\% en 2017; mostrando un comportamiento en tendencia ascendente, cuya función de la línea de tendencia explica $90,31 \%$ de la varianza.

Gráfico 5 Valor agregado total y tasa de crecimiento de la economía en el cantón Machala en la provincia El Oro

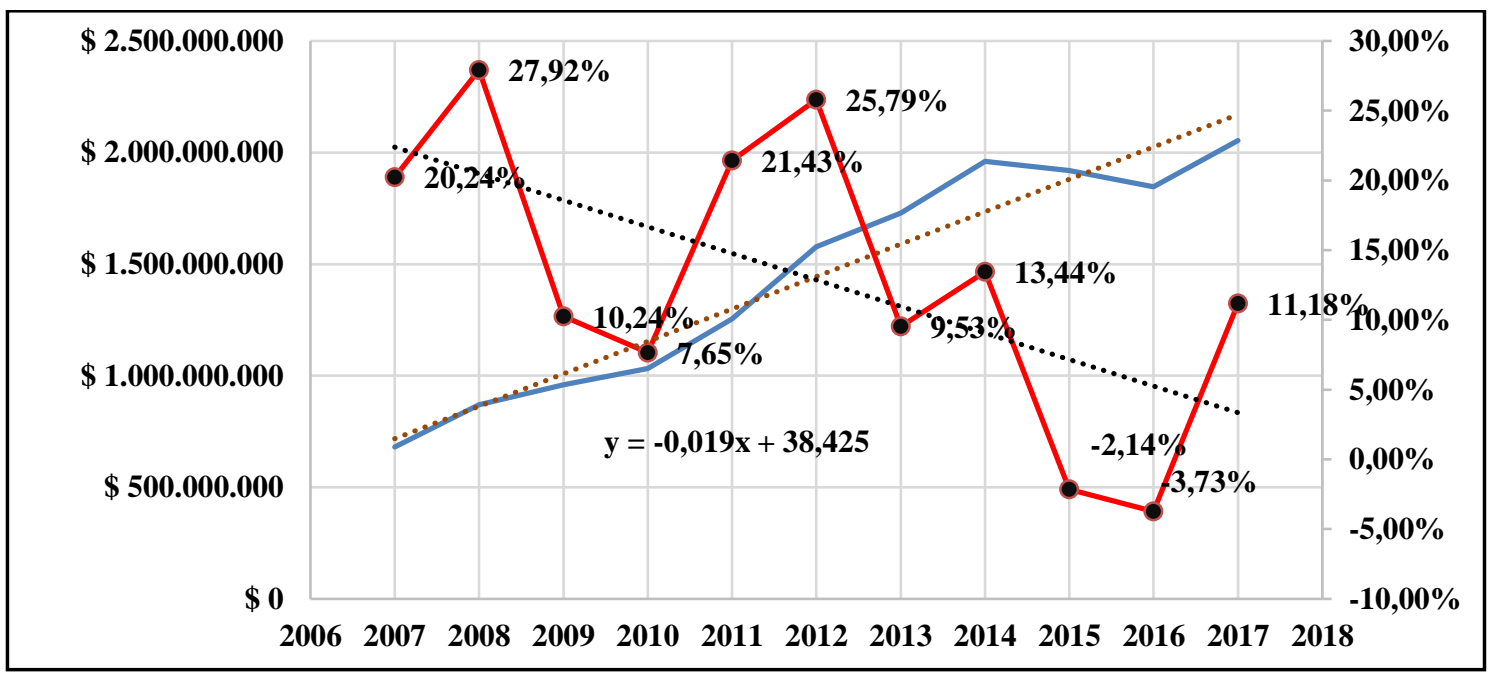

Fuente: Banco Central del Ecuador (2018)

En el gráfico 5 muestra, como a pesar de que en términos absolutos el valor agregado total del cantón Machala presenta una tendencia ascendente, la tasa de crecimiento evidencia una línea de tendencia decreciente con una tasa de crecimiento promedio interanual de -1,9\%; por ello la inestabilidad en la evolución del valor agregado cantonal, se convierte en una limitante para hacer prospectiva de crecimiento positivo del valor agregado cantonal a largo plazo. Al trascender al escenario nacional la realidad no deja de ser distinta. Con una tasa de crecimiento promedio interanual de $-0,78 \%$, como se muestra en el gráfico 6, se observa en ambos gráficos que, mientras el punto de corte de las líneas de tendencia se da en el año 2012 para el escenario local, en el escenario nacional se produjo en el 2008; evidenciando esta última un período más prolongado de caída de la tasa de crecimiento.

En el gráfico 6 también puede observarse una tasa de crecimiento promedio interanual de 4,94\%, la cual resulta al dividir la función marginal entre la función total de la línea de tendencia mostrada para el gráfico 6, la cual explica 93\% de la varianza del valor agregado total cantonal de la economía ecuatoriana. La anterior cifra resultó ser inferior a 6,69\% de la tasa de crecimiento promedio interanual que exhibe el cantón Machala, dejando en evidencia que esta última muestra mejores perspectivas de crecimiento a largo plazo que el escenario nacional. Si se realizan proyecciones para los próximos 10 años, el valor agregado de Machala respecto al valor agregado total de la economía ecuatoriana sería de $2,32 \%$. 
Gráfico 6 Valor agregado cantonal total de la economía y la tasa de crecimiento

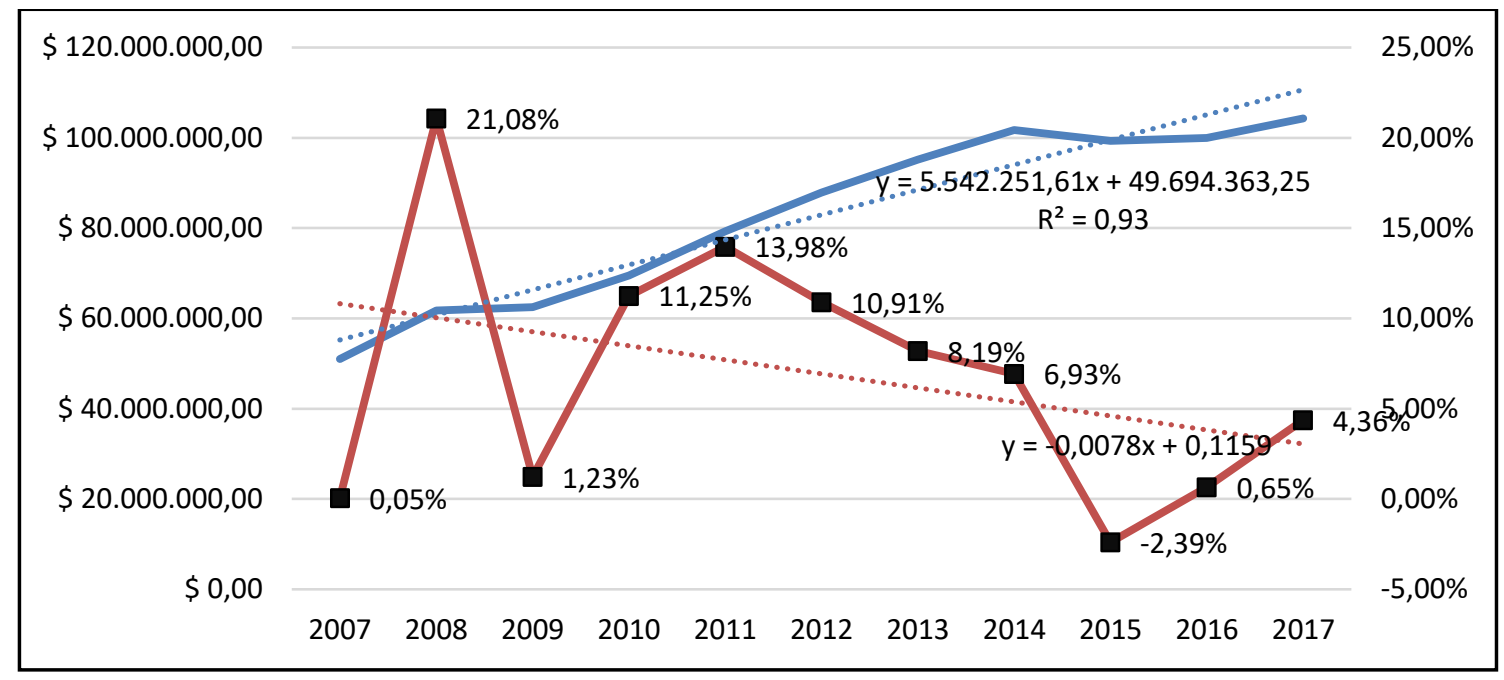

Fuente: Banco Central del Ecuador (2018)

Ante el promedio interanual que exhibe el cantón Machala, se deja en evidencia que esta última muestra mejores perspectivas de crecimiento a largo plazo que el escenario nacional, razón por la cual surge la necesidad de hacer un estudio acerca de la situación de las Pymes en dicha localidad y la importancia suprema que estas tienen por su contribución al valor agregado cantonal, ya que representan según el Instituto Nacional de Estadísticas y Censos del Ecuador (2017), tal como se muestra en la tabla número 1 99,49\% de las 41.429 empresas establecidas en el cantón.

En el gráfico 7 se puede observar el índice relación del (valor agregado bruto/producción total) nacional y del cantón Machala, donde se puede observar como este indicador de productividad, muestra una mayor eficiencia a nivel cantonal que nacional, lo cual se corresponde con la mayor tasa de crecimiento del valor agregado bruto cantonal en términos promedios de 6,69\%; mientras que a nivel nacional la misma es de 4,94; todo ello en buena medida a la presencia una mayor participación de las Pymes en el valor agregado bruto.

Gráfico 7 Índice del Valor agregado bruto/Producción Total Nacional y del Cantón Machala.

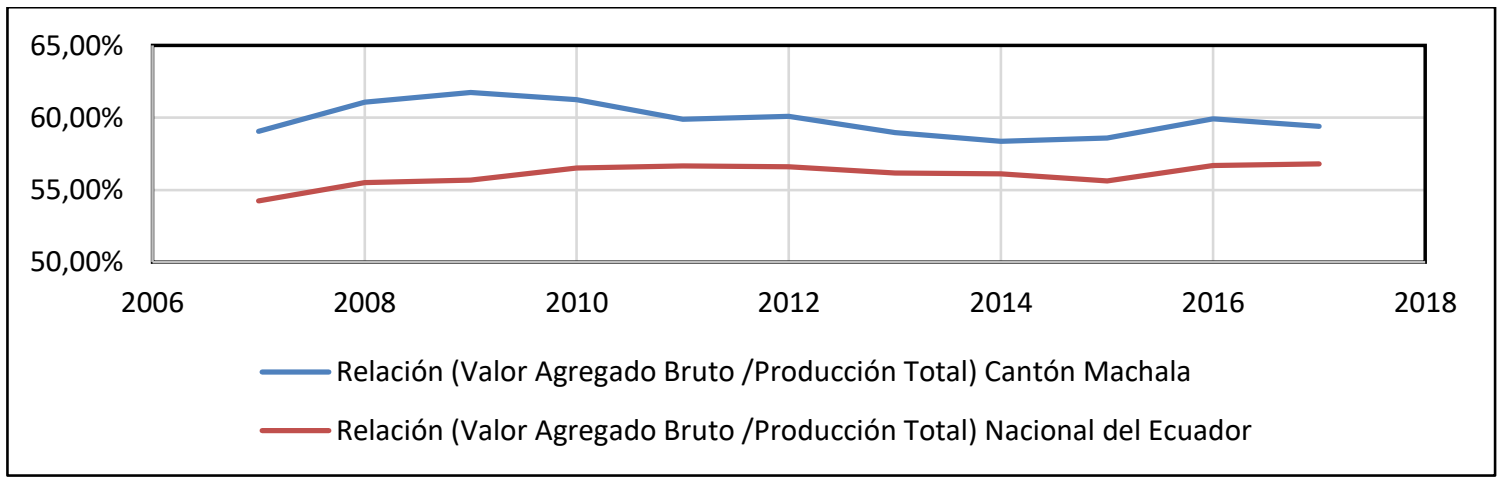

Fuente: Banco Central del Ecuador (2018) 
Tabla 2 Cantón Machala respecto al tamaño de empresas con personal último

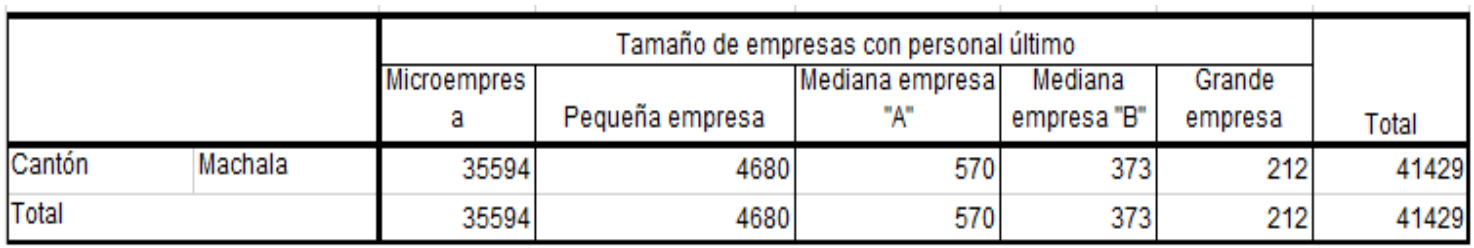

Fuente: Instituto Nacional de Estadísticas y Censos del Ecuador (2017)

En la tabla 2 de acuerdo con los datos mostrados, las microempresas representan 85,92\% del total de las empresas establecidas en el cantón Machala, mientras la pequeña empresa $11,30 \%$, la mediana empresa " $A$ " $1,38 \%$ y la mediana empresa "B" el $0,9 \%$, mientras la grande empresa $0,51 \%$; restante, con lo cual queda en evidencia la importancia de las Pymes en el valor agregado del cantón Machala, ya que de acuerdo a las proyecciones mostradas en la tabla número 3 estas representan $60,18 \%$ de las ventas totales y en similar medida del valor agregado cantonal el cual para el año 2017.

Tabla 3 Cantón Machala respecto al tamaño de empresas con personal último y las ventas totales proyectadas

\begin{tabular}{|c|c|c|c|c|c|}
\hline & & $\begin{array}{l}\text { Promedio des } \\
\text { Ventas totales }\end{array}$ & $\begin{array}{c}\text { N"de } \\
\text { Empreasas }\end{array}$ & $\begin{array}{l}\text { Vental totales } \\
\text { proyectadas }\end{array}$ & $\begin{array}{l}\text { Participación en } \\
\text { las ventas totales }\end{array}$ \\
\hline \multirow{5}{*}{$\begin{array}{c}\text { Tamaño de } \\
\text { omprosas } \\
\text { oon porsonal } \\
\text { ditimo }\end{array}$} & Mioroempresa & $\$ 42.101 .86$ & 36504 & $\$ 1.498 .673 .632 .86$ & $78.07 \%$ \\
\hline & $\begin{array}{l}\text { Pequeña } \\
\text { empreas }\end{array}$ & 9 336.674 .66 & 4680 & $\$ 1.670 .489 .378 .01$ & 18.9496 \\
\hline & $\begin{array}{l}\text { Mediana } \\
\text { empreasa- }\end{array}$ & \&1.406.473.41 & 570 & \$ $801.689 .843,90$ & $0,67 \%$ \\
\hline & $\begin{array}{l}\text { Mediana } \\
\text { empreana " }{ }^{-}\end{array}$ & \$3 $3.001 .078,60$ & 373 & क 1.110 .370 .930 .53 & $13,50 \% 6$ \\
\hline & Crande emprema & F 15.576.103.61 & 212 & q. $.202 .150 .925,09$ & $20,02 \%$ \\
\hline
\end{tabular}

Fuente: Instituto Nacional de Estadísticas y Censos del Ecuador (2017)

En razón de ello, el presente estudio se propone hacer un análisis de una muestra de 127 Pymes establecidas en el cantón Machala como se observa en la tabla número 4, con un 95\% de confianza, un $9 \%$ de error con base al criterio de la máxima varianza, conglomeradas en tres clúster con base al monto total de ventas de cada una de las empresas seleccionadas, de las cuales $47,24 \%$ son medianas empresas " $\mathrm{A}$ " o " $\mathrm{B}$ ", $35,43 \%$ son pequeñas empresas y 17,32\% microempresas; en cuanto a los tres clúster mostrados en la tabla número 4, 41,73\% de las Pymes pertenecen al clúster 1, en igual cuantía clúster 2 ; mientras el $16,53 \%$ restante de las empresas pertenecen al clúster 3; así mismo se detalla la distribución de las Pymes para cada uno de los tres clúster.

Tabla 4 Cantón Machala respecto al tipo de y números de caso de clúster.

\begin{tabular}{|l|l|l|l|l|l|}
\hline \multicolumn{2}{|c|}{} & \multicolumn{2}{|l|}{ Tipo De Empresa } & \multirow{2}{*}{ Total } \\
\cline { 2 - 5 } & Mediana & Micro & Pequeña & \\
\hline & 1 & 29 & 9 & 15 & 53 \\
\hline
\end{tabular}




\begin{tabular}{|l|l|l|l|l|l|}
\hline $\begin{array}{l}\text { Número de } \\
\text { caso de } \\
\text { clúster }\end{array}$ & 2 & 32 & 12 & 19 & 53 \\
\cline { 2 - 7 } & Total & 9 & 1 & 11 & 21 \\
\hline
\end{tabular}

Fuente: Elaboración propia con SPSS ver 22.0

A continuación, en la tabla 5 se muestra a que sectores de la actividad económica en el cantón Machala pertenecen cada uno de los tipos de empresas que forman parte de los clústers. De las cuales $28 \%$ de las Pymes pertenecen al sector agropecuario, $40 \%$ al sector comercio y $32 \%$ restante al sector servicios.

Tabla 5 Tipo de empresa por actividad económica en el cantón Machala y el clúster de pertenencia

\begin{tabular}{|c|c|c|c|c|c|c|}
\hline \multirow{2}{*}{\multicolumn{3}{|c|}{ Número De Caso De Clúster }} & \multicolumn{3}{|c|}{ Actividad Económica } & \multirow{3}{*}{$\begin{array}{l}\text { Total } \\
29\end{array}$} \\
\hline & & & \multirow{2}{*}{$\begin{array}{l}\text { Agropecuaria } \\
12\end{array}$} & \multirow{2}{*}{$\begin{array}{l}\text { Comercio } \\
9\end{array}$} & \multirow{2}{*}{$\begin{array}{l}\text { Servicios } \\
8\end{array}$} & \\
\hline 1 & Tipo & DeMEDIANA & & & & \\
\hline & & MICRO & 1 & 1 & 7 & 9 \\
\hline & & PEQUEÑA & 4 & 5 & 6 & 15 \\
\hline & Total & & 17 & 15 & 21 & 53 \\
\hline 2 & Tipo & DeMEDIANA & 4 & 12 & 6 & 22 \\
\hline & & MICRO & 0 & 7 & 5 & 12 \\
\hline & & PEQUEÑA & 5 & 7 & 7 & 19 \\
\hline & Total & & 9 & 26 & 18 & 53 \\
\hline 3 & Tipo & DeMEDIANA & 4 & 4 & 1 & 9 \\
\hline & Битра & MICRO & 0 & 1 & 0 & 1 \\
\hline & & PEQUEÑA & 6 & 5 & 0 & 11 \\
\hline & Total & & 10 & 10 & 1 & 21 \\
\hline
\end{tabular}

Fuente: Elaboración propia con SPSS ver 22.0 
Para ello, en los tres conglomerados se evaluaron un conjunto de indicadores de carácter administrativo y gerencial a través de un cuestionario de 72 ítems para medir la competitividad de la mediana y pequeña empresa, tales como planificación, comercialización, administración, contabilidad y finanzas, calidad, recurso humano, gestión ambiental y sistemas de información, tal como se muestra en el gráfico número 8. Donde el clúster $\mathrm{N}^{\circ} 1$ en una escala de 1 al 5 tiene una media de efectividad de 4,82 para los indicadores antes señalados; mientras clúster 2 de 4,15 y el clúster 3 de 3,57.

No obstante, en el gráfico 8 se observan diferencias significativas entre el clúster para los indicadores de carácter administrativo y gerencial, tales como planificación, calidad, recurso humano y gestión ambiental; más no así con los indicadores comercialización, administración, contabilidad y finanzas quienes muestran diferencias menos significativas. Razón por la cual, las Pymes que integran los clúster 2 y 3 deben trabajar para que la gestión y proyección de las mismas corresponda a un plan estratégico, el proceso de toma de decisiones involucre a las personas responsables por su ejecución y cumplimiento, cuente con metas de operación y proyecciones financieras medibles y verificables en un plazo determinado, el plan estratégico diseñado debe tener como cimentos un análisis situacional FODA, para determinar las fortalezas y debilidades internas y las oportunidades y amenazas externas que ofrece el entorno, que facilite el establecimiento de objetivos a corto y largo plazo, así como la asignación de recursos para tal fin, todo ello en concordancia con la misión y visión de la organización.

Gráfico 8 Indicadores de carácter administrativo y gerencia

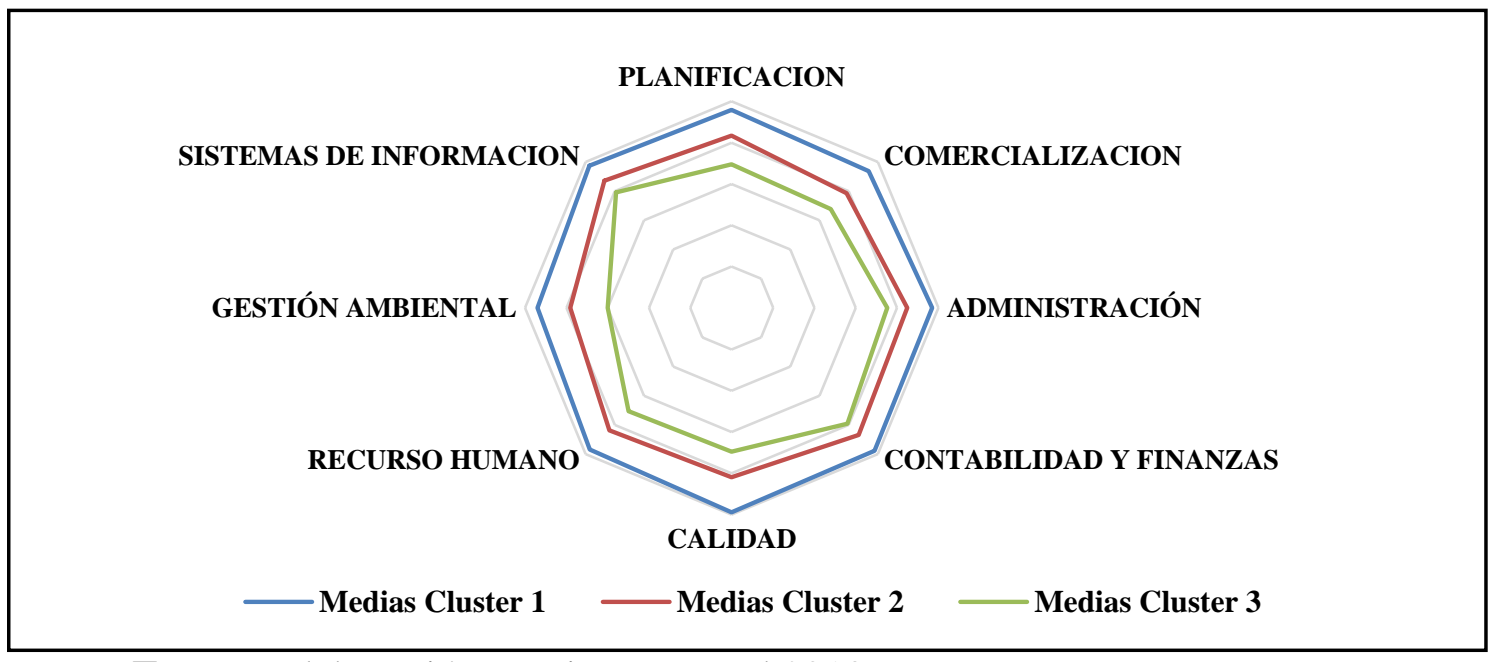

Fuente: Elaboración propia con Excel 2013

Otro indicador a mejorar por parte de las Pymes pertenecientes a los clúster 2 y 3 es la gestión ambiental, haciendo más eficiente su política ambiental, considerando los aspectos ambientales en la planificación de la empresa; midiendo el desempeño ambiental frente a las metas e indicadores seleccionados; reformulando las estrategias y medidas para prevenir, mitigar y corregir los impactos ambientales que genera el proceso productivo tomando en consideración las regulaciones ambientales; introduciendo tecnologías ecoeficientes y limpias; capacitando a los trabajadores en temas ambientales; 
documentando las tareas, responsabilidades, competencias y procedimientos específicos que aseguren el cumplimiento de las normas ambientales, tanto internas como externas; minimizando el consumo de energía, agua y materias primas contaminantes mediante la mejora de sus procesos productivos, el reciclaje, la sustitución de insumos, el mantenimiento preventivo y el uso de otras tecnologías.

Así mismo otro indicador a mejorar es recursos humanos por parte del conglomerado de Pymes es el aspecto atinente a recursos humanos, definiendo claramente la políticas y procedimientos en la búsqueda, selección y contratación de sus trabajadores; estableciendo las competencias que la persona debe poseer basado en un estudio del puesto de trabajo que se va a ocupar (descripción de las tareas, las especificaciones humanas y los niveles de desempeño requerido), para lo cual se requiere de la aplicación de pruebas de conocimiento o capacidad de valoración de las aptitudes y actitudes y de personalidad por personas idóneas para realizarlas; realización de procesos de inducción para los nuevos trabajadores y de re-inducción para los antiguos, programas de entrenamiento en habilidades prácticas y técnicas, formación humana y desarrollo personal para el mejor desempeño de sus colaboradores.

Donde cada puesto de trabajo tiene definida la forma de medir el desempeño de la persona (indicador) lo cual permite su evaluación y elaboración de planes de mejoramiento. Para ello la empresa debe estar alerta para identificar futuros líderes con alto potencial y colaboradores con desempeño superior. Por lo cual es necesario que la planta, los procesos, los equipos y las instalaciones en general están diseñados para procurar un ambiente seguro para el trabajador; aunado una mejora continua en la comunicación entre los diferentes niveles de personal de la compañía (directivos, técnicos, administrativos, otros) se promueve y es ágil y oportuna.

Finalmente el tercer indicador a mejorar es la calidad; para ello es necesario que la empresa tenga una política definida; donde la misma desarrolle a un análisis periódico para identificar los procesos críticos (aquellos que afectan directamente la calidad del producto o servicio); optimizando los métodos de trabajo relacionados con los procesos críticos de la empresa están documentados; automatizando la información de los registros de la aplicación de los procedimientos generales de la empresa es analizada y utilizada como base para el mejoramiento.

Asimismo, estableciendo controles para identificar errores o defectos y sus causas, a la vez que toma acciones inmediatas para corregirlos; diseñando un esquema de acción para ejecutar las acciones correctivas y preventivas necesarias para garantizar la calidad del producto o servicio; fijando una serie de parámetros definidos para la planeación de compra de equipos, materia prima, insumos y demás mercancías; para lo cual es necesario que la empresa mejore y fortalezca la relación con sus proveedores, capacitando a sus colaboradores en temas de calidad y mejoramiento continuo y midiendo el índice de satisfacción del cliente como base para planes de mejora de la organización. 


\section{Conclusiones.}

- Luego de analizados los resultados de la investigación, puede concluirse que el cantón Machala debe continuar profundizando su proceso de diversificación económica, donde actividades productivas que hoy presentan baja participación, pero altas tasas de crecimiento promedio en los últimos 10 años deben continuar al mismo ritmo o acelerarlo para aprovechar en su máxima expresión sus potencialidades, y con ello contribuir a un crecimiento más acelerado del valor agregado total del cantón para seguir aumentando su participación en el valor agregado total cantonal de la economía ecuatoriana.

- Para lograr lo antes expuesto se requiere primeramente que el números de Pymes sigan creciendo, y con ello su participación en el valor agregado total del cantón Machala, para esto es necesario que las mismas, sobre todo las micro y pequeñas empresas mejoren sus procesos de trabajo en lo atinente al manejo de la gestión ambiental, la gestión del recurso humano, la optimización de los procedimientos de control de calidad y el desarrollo de todo una planificación estratégica que responda a un plan estratégico que contemple, la misión y visión de la organización, los objetivos y metas a lograr a corto y largo plazo; el establecimiento de las estrategias ofensivas (fortaleza-oportunidades), supervivencia (debilidades-amenazas), defensiva (fortaleza-amenaza) y reorientación (debilidades-oportunidades), con el fin de lograr los objetivos y metas propuestos previamente.

- Para ello se requiere previamente de la aplicación de un análisis FODA y CAME que permita hacer una valoración situacional de cada empresa, para posteriormente hacer un uso eficiente de la asignación de los recursos financieros necesarios para acometer los planes de acción requeridos para la instrumentación de las estrategias gerenciales anteriormente señaladas.

\section{Referencias Bibliográficas.}

Alesina A y Gavazzi, F. (2006). Goodbye Europa. Cronache de un declino económico e político. Milano: Rizzoli.

Astudillo, S.; Briozzo, A. (2016). Innovación en las Mipymes Manufactureras de Ecuador y Argentina. Semestre Económico, vol. 19, núm. 40, julio-septiembre, 2016, pp. 117-144 Universidad de Medellín, Colombia 
Banco Central del Ecuador (2018). Recuperado de https://contenido.bce.fin.ec/documentos/Estadisticas /SectorReal/CuentasProvinciales/Indicep.htm. Consultado el 13/12/2018.

Bermeo Pazmiño, K. V. \& Saavedra García, M. L. (2018). La competitividad sistémica de la mipyme manufacturera en el nivel micro: caso de la fabricación de muebles de madera, Ecuador. Small Business International Review, 2 (1), 1-15. Recuperado de https://doi.org/10.26784/sbir.v2i1.20. Consultado el 14/12/2018.

BID (Banco Interamericano de Desarrollo), estas son: planificación, comercialización, administración, contabilidad y finanzas, recursos humanos, calidad, gestión ambiental y recursos humanos, (2012, pág. 26-27).

De Gregorio, J. (2012). Macroeconomía Teoría y Políticas. Santiago, Chile: Editorial Pearson-Educación. Segunda Edición. 781 páginas.

Dussel. E. (2001). Un análisis de la competitividad de las exportaciones de prendas de vestir de Centroamérica utilizando los programas y la metodología CAN y MAGIC. México: Naciones Unidas-Cepal.

Gallino, L. (2003). La scomparsa dell’Italia industriale. Torino: Editorial Einaudi.

Ghiringhelli, Cristiano y Pero, Luciano (2010). Le PMI in Italia. Innovazione, strategie, modelli organizzativi. Milano: Editoriale Apogeo, s.r.l.

Hernández, R., Fernández, C. y Baptista, P. (2014). Metodología de la investigación (6ta. ed.). México, DF, México: Ed. Mc Graw Hill-Interamericana. 634 páginas.

Instituto Nacional de Estadísticas y Censos, INEC, (2014). Directorio de empresas. Recuperado en: http://www.ecuadorencifras.gob.ec/directoriodeempresas/ Consultado el 15/12/2014

Instituto Nacional de Estadísticas y Censos, INEC, (2015). Directorio de empresas. Recuperado en: http://www.ecuadorencifras.gob.ec/directoriodeempresas/ Consultado el 15/12/2014

Instituto Nacional de Estadísticas y Censos, INEC, (2017). Directorio de empresas. Recuperado en: http://www.ecuadorencifras.gob.ec/directoriodeempresas/ Consultado el 15/12/2018.

Ministerio de Industrias y Productividad (2013) "Proyecto para el fomento de las MIPYMES Ecuatorianas” elaborado por la secretaría de MIPYMES y Artesanías. 82 páginas.

Nardozzi, G. (2004). Miracolo in declino. L'Italia tra la concorrenza e protezione. Bari: Laterza. 
Onida, F. (2004). Se il piccolo non cresce. Piccole e medie imprese italiane in affano. Bologna: Il Mulino.

Preti, P y Puricelli, M. (2007). L'impresa forte. Un manifesto per le piccole imprese. Milano: Egea.

Rossi, S. (2006). La regina e il cavallo. Quattro mosse contro il declino. Bari: Laterza.

Saavedra García, M. L. (2012). Una propuesta para la determinación de la competitividad en la pyme latinoamericana. Pensamiento y gestión (33), 93-124.

Sainz de Vicuña, J. M. (2010). El plan estratégico en la práctica. ESIC Editorial. 392 páginas.

Sánchez Gómez, M. G. (2008). Cuantificación y generación de valor en la cadena de suministro extendida. Del Blanco editores, 128 páginas.

Superintendencia de Compañías (2012). https://www.supercias.gob.ec/portalscvs/ Consultado el 18/12/2018.

Yance Carvajal, C.; Solís Granda, L.; Burgos Villamar, I y Hermida Hermida, L. (2017). "La importancia de las PYMES en el Ecuador", Revista Observatorio de la Economía Latinoamericana, Ecuador, (junio 2017). En línea: http://www.eumed.net/cursecon/ecolat/ec/2017/pymes-ecuador.html

Zambrano, A. (2011). Planificación estratégica, presupuesto y control de la gestión pública. Universidad Católica Andrés, 376 páginas.

Zamora, G. y Villamar, X. (2011). Caracterización de la PYME en la industria manufacturera del distrito metropolitano de Quito, Quito: Publicaciones Pontificia Universidad Católica del Ecuador (PUCE), 230p.




Para citar el artículo indexado.

Urdaneta A, González A., Luciani L. \& Borgucci E.(2019). Valor Agregado y Nivel Competitividad de las pymes en el Cantón Machala, Ecuador. Revista electrónica Visionario Digital 3(2), 245-265. Recuperado desde: http://visionariodigital.org

\section{Ciencia \\ Ligital \\ Editonial}

El artículo que se publica es de exclusiva responsabilidad de los autores y no necesariamente reflejan el pensamiento de la Revista Ciencia Digital.

El articulo queda en propiedad de la revista y, por tanto, su publicación parcial y/o total en otro medio tiene que ser autorizado por el director de la Revista Ciencia Digital.
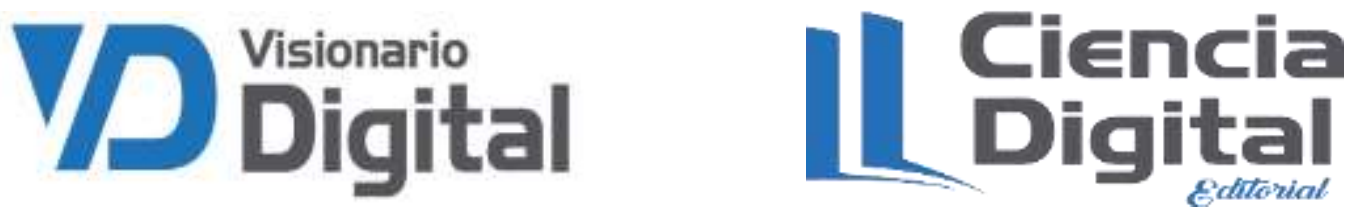